\section{POS0521 FACTORS ASSOCIATED WITH SUBCLINICAL ATHEROSCLEROSIS IN PATIENTS WITH RHEUMATOID ARTHRITIS}

N. Mena-Vázquez ${ }^{1}$, F. G. Jiménez-Núñez ${ }^{1}$, M. Rojas-Giménez², L. Cano Garcia ${ }^{1}$, S. Manrique Arija ${ }^{1}$, A. Fernandez-Nebro ${ }^{1} .{ }^{1}$ Instituto de Investigación Biomédica de Málaga (IBIMA). Hospital Regional Universitario de Málaga (HRUM), Reumatología, Málaga, Spain; ${ }^{2}$ Instituto Maimónides de Investigación Biomédica de Córdoba (IMIBIC). UGC de Reumatología, Hospital Universitario Reina Sofía de Córdoba. Reumatología, Córdoba, Spain

Objectives: To describe the prevalence of subclinical atherosclerosis measured as carotid intima-media thickness (cIMT) in patients with rheumatoid arthritis (RA) and to analyze the associated factors.

Methods: We performed an observational study of 60 patients with RA and 60 sex and age-matched controls. Patients with dyslipidemia were excluded. The main variable was the cIMT measured by ultrasound. The maximum cIMT was measured and atheromatous plaque was defined as focal thickening of the arterial wall protruding toward the lumen and measuring $>0.5 \mathrm{~mm}$ or more than $50 \%$ of the neighboring CIMT. The other variables included were clinical and laboratory values, lipid metabolism, the 28-joint Disease Activity Score (DAS28), International Physical Activity Questionnaire (METs) and Adherence to a Mediterranean diet (MEDAS). Descriptive, bivariate and two multivariate models were constructed to identify factors associated with pathologic cIMT in all subjects and other in RA patients.

Results: The baseline characteristics of both groups are shown in Table 1. The RA patients did not have differences in the mean (SD) of maximum cIMT in relation to the controls $(0.77$ [0.1] vs $0.75[0.1] ; p=0.392)$, nor in the number of plaques (16 $[26.7 \%]$ vs 10 [16.7]; $p=0.184)$. The factors associated with maximum clMT in the total sample were male sex $(B=0.182 ; p=0.039)$, age $(B=$ $0.010 ; p<0.001)$, METs $(B=-2.19 ; p=0.008)$, MEDAS $(B=-0.177 ; p=0.038)$; While the factors associated with maximum cIMT in patients with RA were: male sex $(B=0.155 ; p=0.003)$, age $(B=0.005 ; p=0.007)$, MEDAS $(B=-0.022 ; p=$ $0.017)$, DAS28 ( $B=0.036 ; p=0.003)$ and ACPA $(B=0.082 ; p=0.052)$.

Table 1. Baseline characteristics of 60 patients with RA and 60 controls.

\begin{tabular}{|c|c|c|c|}
\hline Variable & Patients $n=60$ & Controls $n=60$ & p-value \\
\hline Age in years, mean (SD) & $54.0(11.1)$ & $54.2(110.4)$ & 0.943 \\
\hline Female sex; $\mathrm{n}(\%)$ & $53(88.3)$ & $51(85.0)$ & 0.591 \\
\hline Smoking & & & 0.300 \\
\hline Never smoked, n (\%) & $27(45.8)$ & $30(54.5)$ & \\
\hline Exsmoker, $\mathrm{n}(\%)$ & $23(39.0)$ & $14(25.5)$ & \\
\hline Active smoker, $\mathrm{n}(\%)$ & $9(15.3)$ & $11(20,0)$ & \\
\hline BMI $\left(\mathrm{kg} / \mathrm{m}^{2}\right)$, mean (SD) & $28.0(5.5)$ & $27.3(4.9)$ & 0.540 \\
\hline MET-minute, median (IQR) & $533.2(605.1)$ & $809(716.9)$ & 0.028 \\
\hline MEDAS, median (IQR) & $9.4(1.8)$ & $9.1(2.1)$ & 0.349 \\
\hline Progression of RA, months, mean (SD) & $119.7(84.1-170.5)$ & - & - \\
\hline Diagnostic delay, months, median (IQR) & $5.7(5.1-14.4)$ & - & - \\
\hline Erosions, n (\%) & $25(43.1)$ & - & - \\
\hline $\mathrm{RF}>10, \mathrm{n}(\%)$ & $45(75.0)$ & $0(0.0)$ & $<0,001$ \\
\hline ACPA >20, n (\%) & $48(80.0)$ & $0(0,0)$ & $<0,001$ \\
\hline High-sensitivity CRP (mg/dl), median (IQR) & $8.1(4.2)$ & $2.0(4.5)$ & 0.009 \\
\hline ESR $(\mathrm{mm} / \mathrm{h})$, median (IQR) & $21.1(16.6)$ & $13.9(12.3)$ & 0.008 \\
\hline DAS28 at protocol, mean (SD) & $3.1(2.2-4.2)$ & - & - \\
\hline Synthetic DMARDs, n (\%) & $52(88.1)$ & - & - \\
\hline Methotrexate, n (\%) & $36(61.0)$ & - & - \\
\hline Leflunomide, n (\%) & $6(10.2)$ & - & - \\
\hline Sulfasalazine, $n(\%)$ & $7(11.9)$ & - & - \\
\hline Hydroxychloroquine, n (\%) & $4(6.8)$ & & \\
\hline Biologic DMARDs, n (\%) & $32(54.2)$ & - & - \\
\hline Anti TNF- $\alpha, \mathrm{n}(\%)$ & $23(39.0)$ & - & - \\
\hline Jak inhibitor, n (\%) & $1(1.7)$ & - & - \\
\hline Anti-IL-6, n (\%) & $6(10.2)$ & - & - \\
\hline Abatacept, n (\%) & $1(1.7)$ & - & - \\
\hline
\end{tabular}

Abbreviations: RA, rheumatoid arthritis; ACPA, anti-citrullinated peptide antibodies; $R F$ rheumatoid factor; SD, standard deviation; MEDAS: Mediterranean Diet Adherence Survey; DAS28, 28-joint Disease Activity Score; CRP, C-reactive protein; ESR, erythrocyte sedimentation rate; DMARD, disease-modifying antirheumatic drug; IL-6, interleukin 6; Anti TNF, anti-tumor necrosis factor.

Conclusion: In patients with well-controlled established RA, subclinical atherosclerosis is associated, in addition to sex, age, and mediterranean diet, with inflammatory activity and ACPA value.

Acknowledgements: Grant for Medical Researchers from "Fundación Española de Reumatología" 2019

Grant from "Fundación Española de Reumatología" 2018 for non-funded projects. Disclosure of Interests: None declared

DOI: 10.1136/annrheumdis-2021-eular.1561

\section{POS0522 PREVALENCE OF BRONCHIECTASIS IN RHEUMATOID ARTHRITIS: A SYSTEMATIC REVIEW AND META-ANALYSIS}

L. Martin ${ }^{1}$, L. Prisco ${ }^{1}$, W. Huang ${ }^{1}$, G. Mcdermott ${ }^{1}$, N. Shadick', T. Doyle ${ }^{2}$ J. Sparks ${ }^{1} .{ }^{1}$ Brigham and Women's Hospital, Rheumatology, Boston, United States of America; ${ }^{2}$ Brigham and Women's Hospital, Pulmonology, Boston, United States of America

Background: Bronchiectasis is a known extra-articular manifestation of rheumatoid arthritis (RA) and can lead to decreased quality of life as well as increased risk for infection and mortality. Understanding the burden of bronchiectasis in RA may lead to a better understanding of pathogenesis and improved management. We performed a systematic review and meta-analysis to determine the prevalence of bronchiectasis in RA.

Objectives: We investigated the prevalence of RA-related bronchiectasis (RABR) using a systematic review and meta-analysis.

Methods: We followed the PRISMA-P 2015 guideline for systematic reviews and registered this analysis (ID\#199080) on PROSPERO. We queried PubMed and EMBASE databases using the search strategy "rheumatoid arthritis; AND; bronchiectasis" as of July 31, 2020. The inclusion and exclusion criteria were assessed for study eligibility by two independent abstractors. Exclusion criteria included: (1) non-primary literature (i.e., review articles, editorials); (2) case reports involving less than 5 patients; (3) published in a language other than English; (4) did not relate to both RA and bronchiectasis; and (5) studies not involving humans (e.g., mouse models) After the initial screen, we conducted a full text review to verify that inclusion criteria were met: (1) reported frequency of RA-BR and denominator of all RA patients in the study sample. Data including type of study design, method of RA-BR detection, and RA characteristics were extracted by two independent abstractors. We performed meta-analyses using random effects models to estimate prevalence of RA-BR among RA overall and restricted to retrospective or prospective studies.

Results: Out of a total of 208 studies, 37 studies were identified that reported frequency of RA-BR among RA. The included studies had heterogeneous methods to identify RA-BR that were based on either clinical or research chest computed tomography $(\mathrm{CT})$ imaging and had varying methods to adjudicate images. Some studies focused on patients with respiratory symptoms or suspected RA-associated interstitial lung disease (RA-ILD). There were a total of 8,646 patients with $\mathrm{RA}$, and 612 were identified as having RA-BR. The pooled overall prevalence of RA-BR in the random effects meta-analysis was $18.2 \%(95 \% \mathrm{Cl} 13.3-23.7 \%$, Figure 1). Among prospective studies $(n=24)$, the prevalence of RA-BR in the meta-analysis was $20.7 \%(95 \% \mathrm{Cl} 14.7-27.4 \%)$. Among retrospective studies $(n=13)$ reporting RA-BR, the prevalence was $14.5 \%$ (95\% Cl 7.2-23.7\%). Prevalence was lowest in retrospective studies where RA-BR was identified through clinical care (e.g., two large retrospective studies that investigated 4,000 and 1,129 RA patients reported RA-BR prevalence of $0.6 \%$ and $2.7 \%$, respectively). The two largest prospective studies that incorporated a research protocol performing chest CT imaging on all enrolled patients investigated 150 and 332 patients with RA and reported a RA-BR prevalence of $8.0 \%$ and $9.6 \%$, respectively. Smaller studies of both study design types generally reported higher prevalence of RA-BR.

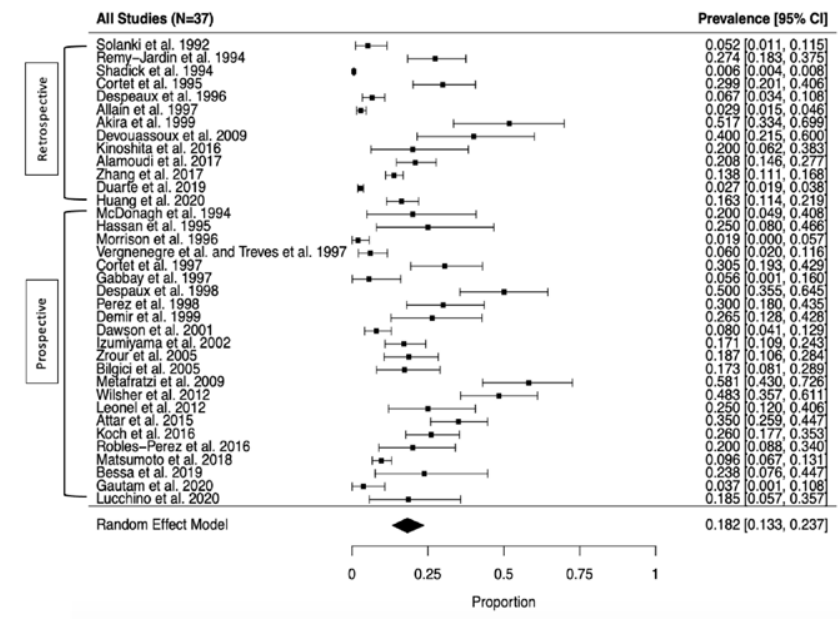

Figure 1. Pooled prevalence of RA-related bronchiectasis in RA among all studies identified $(n=37)$. 
Conclusion: The prevalence of RA-BR in this systematic review and meta-analysis was $18.2 \%$, emphasizing that bronchiectasis is a common extra-articular feature of RA. However, some studies may have identified subclinical RA-BR through research imaging or RA-BR may have been secondary to RA-ILD. Future studies should standardize methods to identify RA-BR cases and investigate the natural history and clinical course given the relatively high prevalence that we report.

Disclosure of Interests: Lily Martin: None declared, Lauren Prisco: None declared, Weixing Huang: None declared, Gregory McDermott: None declared, Nancy Shadick Consultant of: Consultant < 5K Bristol-Myers Squibb, Grant/ research support from: BMS Amgen Lilly, Mallinckrodt, and Sanofi, Tracy Doyle Consultant of: Boehringer Ingelheim $(<5 \mathrm{~K})$, Grant/research support from: Bristol Myers Squibb and Genentech, Jeffrey Sparks Consultant of: Bristol-Myers Squibb, Gilead, Inova Diagnostics, Optum, and Pfizer, Grant/research support from: Bristol-Myers Squibb

DOI: 10.1136/annrheumdis-2021-eular.1650

\section{\begin{tabular}{|l|l}
\hline POS0523 RISK OF CARDIOVASCULAR DISEASE IN NEWLY \\
\hline
\end{tabular} DIAGNOSED RHEUMATOID ARTHRITIS: A CURRENT RISK ASSESSMENT}

R. Raadsen ${ }^{1}$, R. Hansildaar ${ }^{1}$, M. Heslinga ${ }^{1,2}$, A. W. van Kuijk ${ }^{1}$ M. Nurmohamed ${ }^{1,2} .{ }^{1}$ Amsterdam Rheumatology and Immunology Center, Location Reade, Rheumatology, Amsterdam, Netherlands; ${ }^{2}$ Amsterdam

Rheumatology and Immunology Center, Location VU University Medical Center, Rheumatology, Amsterdam, Netherlands

Background: Patients with rheumatoid arthritis (RA) are known to have an increased risk of developing, mainly ischemic cardiovascular diseases (CVD). This is partly explained by a higher incidence of traditional CV risk factors as hypertension and/or hypercholesterolemia but also due to the systemic inflammation characteristic to RA. RA is considered an independent $\mathrm{CV}$ risk by National and International CVD risk management guidelines, and therefore CVD risk screening is recommended. However, the implementation of CV risk management is often poorly performed in daily clinical practice.

Objectives: The aim of the current study was to investigate the cardiovascular disease risk of newly diagnosed RA patients, and review if and to what extent implementation of CVD risk management had previously taken place.

Methods: Every newly diagnosed RA patient in a large Dutch outpatient rheumatology clinic had CV screening within their first year post-diagnosis during a regularly scheduled visit, where among others smoking habits, blood pressure and lipid profile were measured. A 10-year cardiovascular risk was calculated using a modified SCORE calculator from www.u-prevent.com, which predicts the risk of myocardial infarction, stroke, heart failure, peripheral arterial disease and CV death based on several CV risk factors. To account for RA as a risk factor, the calculator multiplies the risk by 1.5 , in accordance with the Dutch CV risk management guideline. The calculated 10-year cardiovascular morbidity risk was categorized in low $(<10 \%)$, intermediate $(10-19 \%)$ and high $(\geq 20 \%)$, and the 10 -year mortality risk was also categorized in low $(<1 \%)$, intermediate $(1-4 \%)$ and high $(\geq 5 \%)$.

Results: A total of 53 patients were included up to this point. Of these, the average age was $57( \pm 8.5)$, and $39(74 \%)$ were women. Mean systolic blood pressure was $129( \pm 17.7) \mathrm{mmHg}$, with $38(72 \%)$ patients having hypertension. Mean total cholesterol, LDL and HDL were respectively $5.5( \pm 1.0) \mathrm{mmol}, 3.43( \pm 0.82) \mathrm{mmol}$ and $1.78( \pm 0.54) \mathrm{mmol}$, with 30 patients having hypercholesterolemia. The average calculated 10 -year morbidity risk of all patients was $13 \%$, and the mortality risk was $3 \%$. Distribution of the 10 -year risk in low, intermediate and high is presented in figure 1 . Only one patient reported having previous used medication for hypercholesterolemia, but not at the time of enrollment in this study.

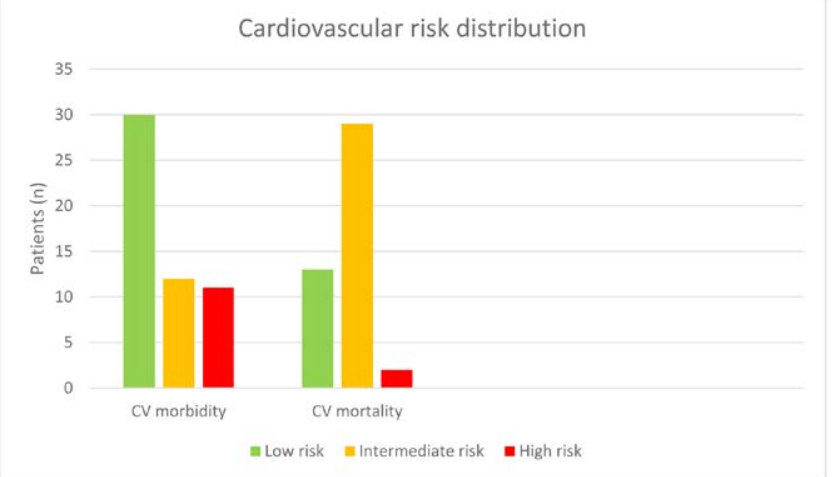

Figure 1. Distribution of 10-year cardiovascular risk in rheumatoid arthritis patients. $\mathrm{CV}=$ cardiovascular
Conclusion: $43 \%$ of newly diagnosed RA patients had an intermediate or high calculated 10 -year CV morbidity risk, and $76 \%$ had an intermediate or high mortality risk. Patients with early RA still often have underdiagnosed and severely undertreated risk factors of CVD, such as hypertension and hypercholesterolemia. Early CV risk assessment and management in patients with RA is critical and needs to be implemented as standard care.

REFERENCES:

[1] Ortec. (2020, November 20). U-prevent. Retreived from U-prevent (u-prevent.nl)

Disclosure of Interests: None declared

DOI: 10.1136/annrheumdis-2021-eular.1734

\section{POS0524 \\ CARDIOVASCULAR DISEASE RISK IN INFLAMMATORY ARTHRITIS PATIENTS STILL SUBSTANTIALLY ELEVATED IN 2020}

R. Raadsen ${ }^{1}$, F. Hooijberg ${ }^{1}$, L. Boekel ${ }^{1}$, E. Vogelzang ${ }^{2}$, M. Leeuw ${ }^{1}$, R. van Vollenhoven $^{3}$, W. Lems ${ }^{1,4}$, G. J. Wolbink ${ }^{1}$, A. W. van Kuijk ${ }^{1}$, M. Nurmohamed ${ }^{1,4}$ ${ }^{1}$ Amsterdam Rheumatology and Immunology Center, location Reade, Rheumatology, Amsterdam, Netherlands; ${ }^{2}$ Amsterdam University Medical Center, Vanderbilt University Medical Center, Medical Microbiology, Amsterdam, Netherlands; ${ }^{3}$ Amsterdam Rheumatology and Immunology Center, Location Academic Medical Center, Rheumatology, Amsterdam, Netherlands; ${ }^{4}$ Amsterdam Rheumatology and Immunology Center, Location VU University Medical Center, Rheumatology, Amsterdam, Netherlands

Background: Patients with inflammatory rheumatic diseases such as rheu matoid arthritis (RA), psoriatic arthritis (PsA) and ankylosing spondylitis (AS) are at a higher risk for developing cardiovascular diseases (CVD) than the general population. This increased risk is partly due to a higher incidence of traditional cardiovascular (CV) risk factors, such as hypertension and dyslipidemia, and partly due to the underlying systemic inflammation. During the past two decades, the burden of the systemic inflammation has been reduced by more efficacious anti-inflammatory treatment, which somewhat attenuated the increased $\mathrm{CV}$ risk of rheumatic patients. However, it remains important to monitor the effects of these new treatment strategies on the prevalence of CVD in patients with a rheumatic disease in systematically controlled cohorts.

Objectives: The aim of the current report was to evaluate whether the CV risk of patients with inflammatory rheumatic diseases still differs from the general population, despite advances In anti-rheumatic treatment strategies.

Methods: In March 2020, all adult patients with an inflammatory rheumatic disease from the Amsterdam Rheumatology and Immunology Center, location "Reade" were systematically asked to participate in a prospective cohort study. The primary aim of this study was to monitor the impact of the COVID 19 pandemic on patients with inflammatory rheumatic diseases compared to age and sex matched healthy controls. Between April 26, 2020 and May 27, 2020, participants completed the first online questionnaire of the study. Amongst others, information on demographic data, including CV comorbidities and risk factors, and medication use was collected. The baseline characteristics and prevalence of CVD were compared between RA, PsA or AS and healthy controls.

Results: In total, 1455 consecutive patients with an inflammatory rheumatic disease (979 RA patients, 261 PsA patients and 215 AS patients), and 414 healthy controls completed the first questionnaire, as shown in table 1. CV comorbidities were more frequently reported in RA, PsA and AS patients compared to healthy controls; $107(11 \%), 28(11 \%)$ and $22(10 \%)$ compared to 30 (7\%), respectively.

Table 1. Biological DMARD usage in RA, PsA and AS patients

\begin{tabular}{lccccc}
\hline Patient characteristics & $\begin{array}{c}\text { All patients } \\
(\mathbf{n = 1 4 5 5 )}\end{array}$ & $\begin{array}{c}\text { RA } \\
(\mathbf{n}=\mathbf{9 7 9})\end{array}$ & $\begin{array}{c}\text { PsA } \\
(\mathbf{N}=\mathbf{2 6 1})\end{array}$ & $\begin{array}{c}\text { AS } \\
(\mathbf{n}=\mathbf{2 1 5})\end{array}$ & $\begin{array}{c}\text { Controls } \\
(\mathbf{n}=\mathbf{4 1 4})\end{array}$ \\
\hline Mean age - yr & $55 \pm 13$ & $58 \pm 12$ & $55 \pm 13$ & $48 \pm 13$ & $53 \pm 13$ \\
Female sex - no (\%) & $934(64)$ & $728(74)$ & $119(46)$ & $87(41)$ & $298(72)$ \\
BMI (IQR) & $25(23-28)$ & $25(22-28)$ & $26(24-30)$ & $25(22-28)$ & $24(22-27)$ \\
Smoking - no (\%) & $178(12)$ & $126(13)$ & $17(7)$ & $35(16)$ & $34(8)$ \\
Cardiovascular disease - no (\%) & $157(11)$ & $107(11)$ & $28(11)$ & $22(10)$ & $30(7)$ \\
Rheumatic medication - no (\%) & & & & & \\
csDMARDs & $877(60)$ & $712(73)$ & $148(57)$ & $17(8)$ & N.A. \\
Oral glucocorticoids & $161(11)$ & $139(14)$ & $17(7)$ & $5(2)$ & $2(0.4)$ \\
TNF inhibitor & $563(39)$ & $336(34)$ & $121(46)$ & $106(49)$ & N.A. \\
IL-6 inhibitor & $19(1)$ & $19(2)$ & 0 & 0 & N.A. \\
IL-17 inhibitor & $17(1)$ & $2(0.2)$ & $7(3)$ & $8(4)$ & N.A. \\
\end{tabular}

Table 1. Baseline characteristics. Values are displayed as mean \pm standard deviation (SD), median with interquartile range (IQR) or frequencies with percentages $(\%)$. RA = rheumatoid arthritis, $P S A=$ psoriatic arthritis, $A S=$ ankylosing spondylitis, $B M I=$ body mass index, TNF $=$ anti-tumor necrosis factor $1 \mathrm{~L}=$ interleukin. 